\title{
Comparison between Direct Electrical and Optogenetic Subthalamic Nucleus Stimulation
}

\author{
Ruben Schoeters $^{1 *}$, Thomas Tarnaud ${ }^{1}$, Wout Joseph ${ }^{1}$, Luc Martens ${ }^{1}$, Robrecht Raedt ${ }^{2}$, Emmeric Tanghe ${ }^{1}$ \\ ${ }^{1}$ Department of Information Technology, Ghent University/IMEC, 9052 Zwijnaarde, Belgium, *ruben.schoeters@ ugent.be \\ ${ }^{2}$ Laboratory for Clinical and Experimental Neurophysiology, Neurobiology and Neuropsychology (LCEN3), Department of \\ Neurology, Ghent University, Belgium
}

\begin{abstract}
Subthalamic nucleus deep brain stimulation is a treatment for Parkinson's disease. In this study, a computational model of a plateau-potential generating subthalamic nucleus neuron (Otsuka-model) and a four-state ChR2(H134R) model (Williams-model) are combined, in order to compare electrical and optogenetic neuromodulation capabilities. The impact of the stimulation modality (optogenetic or electric) on firing rates, strength-duration curves and action potential shape is investigated. First, in contrast to electrical stimulation, mean instantaneous firing rates saturate for optical stimulation at intensities higher than $0.1 \mathrm{~W} / \mathrm{cm}^{2}$. Second, rheobase and chronaxie are $175 \%$ and $9.6 \%$ larger in optogenetic stimulation compared to electrical stimulation, respectively. Third, action potential shape is not significantly impacted by the neurostimulation modality.
\end{abstract}

Keywords-Optogenetics, subthalamic nucleus, computational modeling, Otsuka-model, Channelrhodopsin-2 (H134R)

\section{INTRODUCTION}

Optogenetics is a neuromodulation technique, in which cells are photosensitized by genetically expressing light-sensitive ion channels (opsins). Subsequently, neuronal firing is controlled by exposure to light with high spatial and temporal resolution. As a result, the technique has been used extensively to answer fundamental research questions, e.g. in sleep research and to investigate memory formation and fear conditioning. Furthermore, optogenetics might improve the treatment of neurological disorders, e.g. Parkinson's disease [1-2].

In subthalamic nucleus deep brain stimulation (STN DBS), electrical currents are applied to the STN, as a treatment for Parkinson's disease. However, current spread to neighbouring nuclei and fibre tracts can result in stimulation-induced sideeffects [3], such as facial contractions, ocular deviations, mood and cognitive changes... Consequentially, due to its superior spatial accuracy, optogenetics might improve STN DBS.

In this study, we compare the neuromodulation capabilities of both electrical and optogenetic stimulation of the STN.

\section{MethodS}

The Otsuka-model is a single-compartment model, used to simulate a plateau-potential generating STN-neuron [4]. For simulations of optogenetic neuromodulation, a modification of the four-state ChR2(H134R) model of Williams et al. is used [5]. The models are implemented in Matlab and are simulated
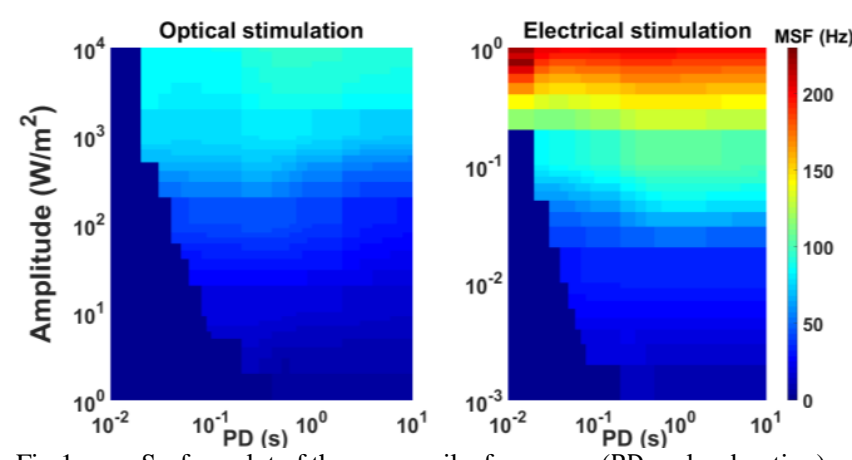

Fig 1 Surface plot of the mean spike frequency (PD: pulse duration). (Left) optical stimulation. (Right) electrical stimulation. Dark blue area in the lower left corner indicates the absence of two subsequent spikes during the pulse.

with the ode113 and ode15s functions, for electrical and optogenetic stimulation respectively [6]. A maximal discretization step $\Delta t=\max \left(10 \mu \mathrm{s}, \frac{\mathrm{PD}}{20}\right)$ is imposed, with PD the duration of the applied rectangular light or current pulse. Tolerances are set to $10^{-10}$ for optical stimulation, and to $10^{-6}$ and $10^{-3}$ (absolute and relative tolerance, respectively) for electrical stimulation.

\section{RESULTS}

The mean spiking frequency (MSF) during the applied pulse is shown in Fig. 1. The MSF is defined as:

$$
\mathrm{MSF}=\frac{n}{t_{n+1}-t_{1}} .
$$

Here, $n$ is the number of interspike intervals and $t_{i}$ is the time of the $i$ th spike. A maximal MSF of $95 \mathrm{~Hz}$ and $230 \mathrm{~Hz}$ is obtained for optical $\left(\mathrm{I}=1 \mathrm{~W} / \mathrm{cm}^{2}\right)$ and electrical stimulation $\left(\mathrm{I}=1 \mathrm{~A} / \mathrm{m}^{2}\right)$, respectively. We note that both MSF-maps match, for intensities up to $\mathrm{I}=0.1 \mathrm{~A} / \mathrm{m}^{2}$.

The mean instantaneous firing rate during the applied pulse, shown in Fig. 2, is defined as:

$$
\mathrm{FR}_{\mathrm{ISI}, \mathrm{DP}}=\sum_{\mathrm{i}=1}^{\mathrm{n}} \frac{1}{\mathrm{I}}
$$

Here, $n$ is the number of interspike intervals and $\mathrm{I}_{i}$ refers to the $i$ th interspike interval. First, we observe that, in contrast with electrical stimulation, mean instantaneous firing rate curves will saturate above $0.1 \mathrm{~W} / \mathrm{cm}^{2}$ for optogenetic stimulation. Second, the standard deviation of the instantaneous firing rate distribution is small, which is indicative of regular spiking in 


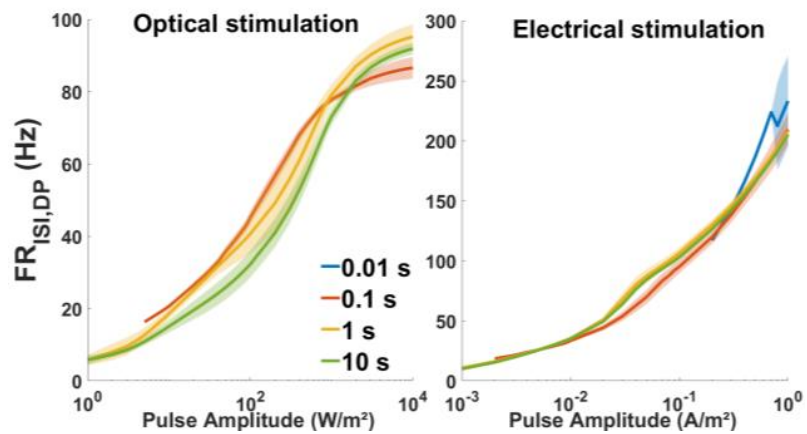

Fig. 2 AP firing frequency versus pulse amplitude and duration. Mean instantaneous frequency \pm standard deviation (shaded area) with respect to pulse amplitudes, calculated over pulse duration for optical and electrical stimulation, respectively.

the absence of noise. The same conclusions can be drawn from Fig. 3 (instantaneous firing rate as function of time).

Strength-duration (SD) curves are shown in Fig. 4 and are fit with the Hill-Lapicque equation. The SD-plots are defined similarly to [7]: for optogenetic stimulation a threshold average stimulating current $\mathrm{I}_{\mathrm{th}, \text { avg }}$ is defined as:

$$
\mathrm{I}_{\mathrm{th}, \mathrm{avg}}=\frac{Q_{13}}{\min (\mathrm{PD}, 13 \mathrm{~ms})} \text {. }
$$

Here, $Q_{13}$ is the injected ChR2-charge, during $13 \mathrm{~ms}$ after stimulus onset. The threshold is defined as the minimum average stimulating current, that results in an action potential (AP) latency smaller than $10 \mathrm{~ms}$.

The rheobase and chronaxie are $175 \%$ and $9.6 \%$ higher for optogenetic stimulation compared to electrical stimulation, implying that electrical stimulation is more efficient for all pulse durations. This is expected from the ChR2 dynamics: while the electrical applied pulse is modeled with negligible rise and decay time, the light-triggered activation of the ChR2current is a kinetic process with time constant $>1 \mathrm{~ms}$.
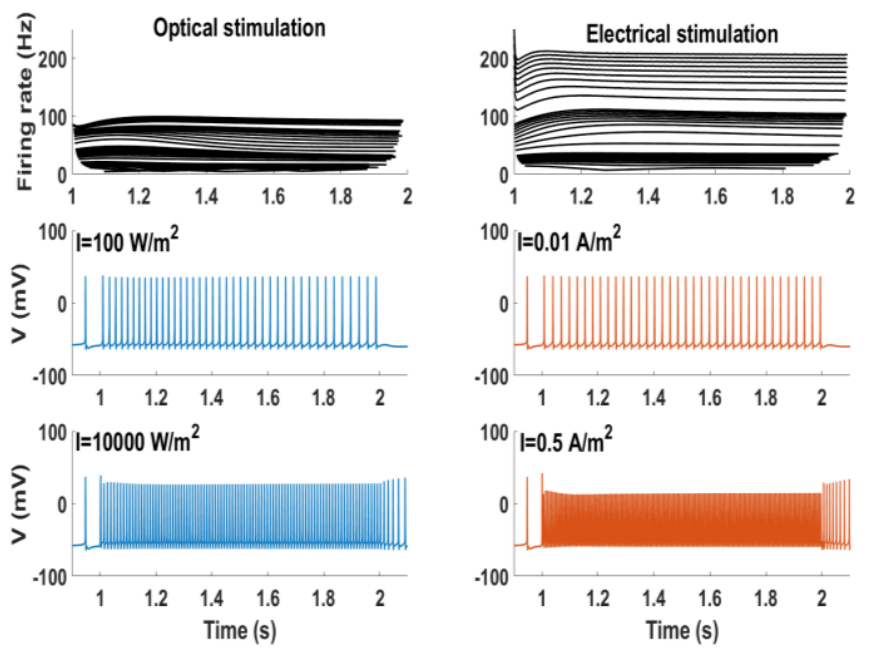

Fig. 3 The instantaneous firing rate during a $1 s$ pulse. (Top) The instantaneous firing rate for the whole amplitude set during a $1 \mathrm{~s}$ pulse. (Middle) the membrane potential for optical stimulation with amplitudes $100 \mathrm{~W} / \mathrm{m}^{2}$ and $10000 \mathrm{~W} / \mathrm{m}^{2}$, respectively. (Bottom) the membrane potential for electrical stimulation with amplitudes $0.01 \mathrm{~A} / \mathrm{m}^{2}$ and $0.5 \mathrm{~A} / \mathrm{m}^{2}$, respectively.

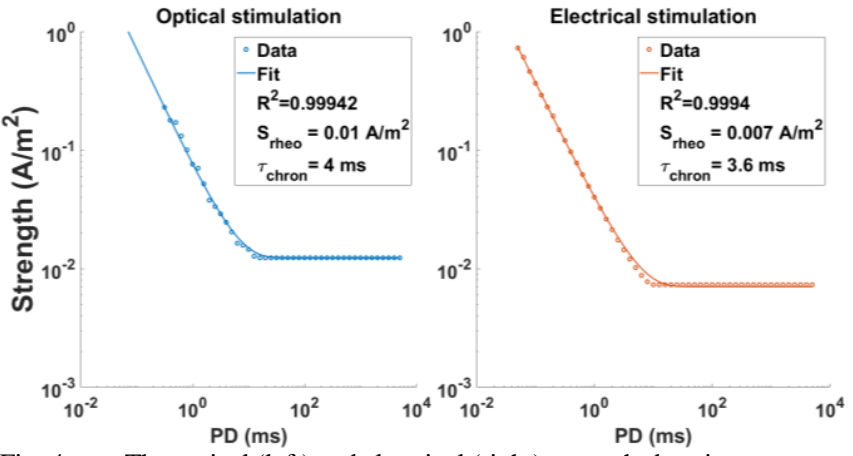

Fig. 4 The optical (left) and electrical (right) strength-duration curves. Strength-duration curves are fit with the Hill-Lapicque equation.

Finally, the AP shape is not significantly impacted by the choice for optogenetic stimulation, due to the voltage dependency of the ChR2-current and because the optogenetic current is relatively small, w.r.t. to the total membrane current.

We conclude that while optogenetic stimulation has higher spatial resolution, electrical stimulation is more efficient and results in a larger firing rate dynamic range (no saturation).

\section{CONCLUSION}

We compared electrical and optogenetic neuromodulation of the subthalamic nucleus in terms of the firing rate, strengthduration curves, and AP shape. This study will be the basis for future work on the potential applicability of optogenetics to deep brain stimulation.

\section{ACKNOWLEDGMENT}

This research was funded by the FWO-project G046816N. T. Tarnaud is a PhD Fellow of the FWO-V (SB) (Research Foundation Flanders, Belgium). E. Tanghe is a Post-Doctoral Fellow of the FWO-V. This publication is based upon work from COST Action EMF-MED, supported by COST (European Cooperation in Science and Technology).

\section{REFERENCES}

[1] E. A. Pama, S. L. Colzato, and B. Hommel. "Optogenetics as a neuromodulation tool in cognitive neuroscience," Frontiers in psychology, vol. 4, pp. 1-4, 2013.

[2] M. Häusser, "Optogenetics: the age of light," Nature methods, vol 11, no. 10, pp. 1012-1014, 2014.

[3] W. M. Grill, "Safety considerations for deep brain stimulation: review and analysis," Expert review of medical devices, vol. 2, no. 4, pp. 409-420, 2005 .

[4] T. Otsuka, T. Abe, T. Tsukagawa, and W.-J. Song, "Conductance-based model of the voltage-dependent generation of a plateau potential in subthalamic neurons," Journal of Neurophysiology, vol. 92, no. 1, pp. 255-264, 2004.

[5] J. C. Williams, J. Xu, Z. Lu, A. Klimas, X. Chen, C. M. Ambrosi, I. S. Cohen, and E. Entcheva, "Computational optogenetics: empiricallyderived voltage- and light-sensitive channelrhodopsin-2 model," PLoS Computational Biology, vol. 9, no. 9, pp. 17-19, 2013.

[6] L. F. Shampine, and M. W. Reichelt, "The matlab ode suite," SIAM journal on scientific computing, vol. 18, no. 1, pp. 1-22, 1997.

[7] J. C. Williams, and E. Entcheva, "Optogenetic versus electrical stimulation of human cardiomyocytes: Modeling insights," Biophysical Journal, vol. 108, no. 8, pp. 1934-1945, 2015 